\title{
LARGE RETROPERITONEAL ABSCESS- DIAGNOSTIC DILEMMA BUT SUCCESSFUL MANAGEMENT- A CASE REPORT
}

\author{
Partha Sarathi Mandal1, Sushanta Sarkar², Soumyendranath Mitra ${ }^{3}$
}

1 Visting Surgeon, Department of General Surgery, Kakdwip Super Speciality Hospital, West Bengal, India.

${ }^{2}$ Postgraduate Trainee, Department of Radiodiagnosis, IPGME and R, Kolkata, West Bengal, India.

${ }_{3}^{3}$ Medical Superintendent, Dr. B. N. Basu Memorial Clinic, Apollo Nursing Home, Kolkata, West Bengal, India.

HOW TO CITE THIS ARTICLE: Mandal PS, Sarkar S, Mitra S. Large retroperitoneal abscess- diagnostic dilemma but successful management- a case report. J. Evolution Med. Dent. Sci. 2018;7(24):2912-2915, DOI: 10.14260/jemds/2018/656

\section{PRESENTATION OF CASE \\ History of Present Illness}

A 65-year-old gentleman was admitted on 22-02-2018 with chief complaints of intractable diarrhoea with abdominal distension, fever with low back pain and painful right inguinal swelling with other constitutional symptoms like bodyache, myalgia, lethargy and anorexia- developed over past 3 weeks. Initially, the patient was treated by local physician, but there was no improvement. The patient was a known smoker and alcoholic.

\section{Past History}

No significant past medical and surgical history and no history of previous hospital admission.

\section{Family History}

No history of same illness in family members.

\section{Examination}

General Survey- Patient was sick-looking and dyspnoeic (Respiratory rate -36 breaths per min). Pallor was mild and body temperature was raised $\left(100^{\circ} \mathrm{F}\right)$. He had mild icterus and bilateral pedal oedema. But he had no cyanosis or clubbing. Cervical lymph nodes were not palpable. On admission, he had tachycardia (120 bpm, regular) and BP was $150 / 90 \mathrm{mmHg}$ and Sp02- $88 \%$ on room air.

\section{Systemic Examination \\ Abdomen}

1. Distended, but there was no shifting dullness.

2. Diffuse abdominal tenderness was present, although tenderness mostly elicited at McBurney's point.

3. A tender diffuse swelling was seen in right-sided inguinal region extending upto the root of scrotum. The upper margin was not defined, and it was irreducible and there was no cough impulse.

4. Scrotal examination- both testes were normal.

5. Rectal examination- multiple prolapsed external haemorrhoids seen. No blood was on tip of finger and no mass was felt.

'Financial or Other Competing Interest': None.

Submission 30-04-2018, Peer Review 25-05-2018,

Acceptance 31-05-2018, Published 11-06-2018.

Corresponding Author:

Dr. Partha Sarathi Mandal,

Poiladanga, Behind Himalayan Sweets,

P. O. Madarat,

P. S. Baruipur-743610,

West Bengal, India.

E-mail: hemendra_partha@yahoo.co.in

DOI: $10.14260 /$ jemds $/ 2018 / 656$

\section{Chest}

Coarse crepitations were audible over right hemithorax.

CVS

- S1 and S2- audible and normal.

- No murmur was present.

\section{INVESTIGATIONS}

Blood- Haemoglobin- 10.1 gm\%

Total Leucocyte Count-19660/cmm (P87L10M1E2)

Peripheral Smear-Toxic granules seen.

CRP-258 mg/L (upto $6 \mathrm{mg} / \mathrm{L}$ )

Urea-48 mg\%, Creatinine- $1.2 \mathrm{mg} \%$

Sodium-132 mEq/L, Potassium- $3.1 \mathrm{mEq} / \mathrm{L}$

HbA1c-5.9\%

LFT-Bilirubin- $3.95 \mathrm{mg} \%$ (Direct- $1.9 \mathrm{mg} \%$ and Indirect- 2.05 mg\%), SGPT- 28 U/L, SGOT- 44 U/L, Alkaline phosphatase$93 \mathrm{U} / \mathrm{L}$ and Total Protein- $5.66 \mathrm{~g} / \mathrm{dL}$.

HBsAg, Anti-HCV and HIV were non-reactive.

\section{Arterial Blood Gas Analysis}

pH- 7.530 (High), pCO2- $24.5 \mathrm{mmHg}$ (Low), $\mathrm{pO}_{2}-81.1 \mathrm{mmHg}$ (Low), $\mathrm{CHCO}_{3}-20.5 \mathrm{mmol} / \mathrm{L}$ (Low), BE (ECF)- $2.2 \mathrm{mmol} / \mathrm{L}$ (Low), $\mathrm{cSO}_{2}-90 \%$ (Low), Lactate- $1.49 \mathrm{mmol} / \mathrm{L}$ (High).

Aerobic Blood Culture- No growth.

\section{Urine Examination}

- Routine examination- Pus cell- 3 - 4/hpf, RBC- not found, Protein- absent.

- $\quad$ Culture and sensitivity- No growth.

CXR PA View (23-02-2018)

Prominent lung interstitial markings.

USG Whole Abdomen (23-02-2018)

Fatty liver, Prostatomegaly with significant post void residual urine.

\section{CECT Whole Abdomen (26-02-2018)}

Large loculated collection in the deeper aspect of the right lateral abdominal wall extending downwards into the right inguinal canal. Multiple pockets of air are seen within the collection. In the lower abdomen, there is continuation of this collection along the anterior abdominal wall across the midline. The appendix appears thickened and there is also a pocket of air near its tip. There is thickening of the right perinephric fascia with increased density of right perinephric fat suggestive of inflammatory changes. There is tracking of fluid above the posterior aspect of left psoas and 
left obturator seen deep in the pelvis and also in the left thigh. Air is also seen in these areas.

Final impression was- Large extraperitoneal collection of fluid with air in lower abdomen and pelvis as described with probable extraperitoneal rupture of appendicular tip with inflammatory changes seen in the right perinephric areas.

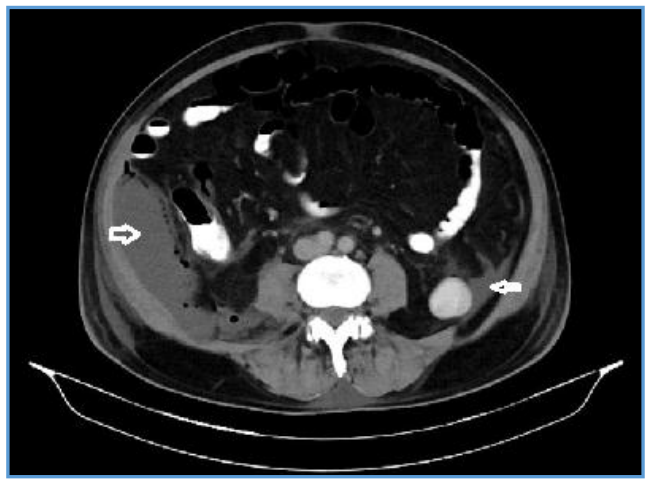

Figure 1. CECT W/A showing both-sided Retroperitoneal Collection (Arrows)

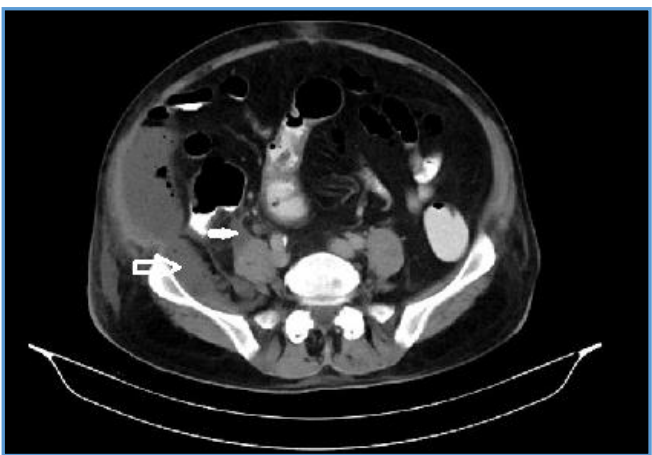

Figure 2. CECT W/A showing Collection Tracking over right-sided Iliacus and Psoas Muscle (Arrows)



Figure 3. CECT W/A showing Collection in Anterior Abdominal Wall and involvement of left Obturator Internus (Arrows)

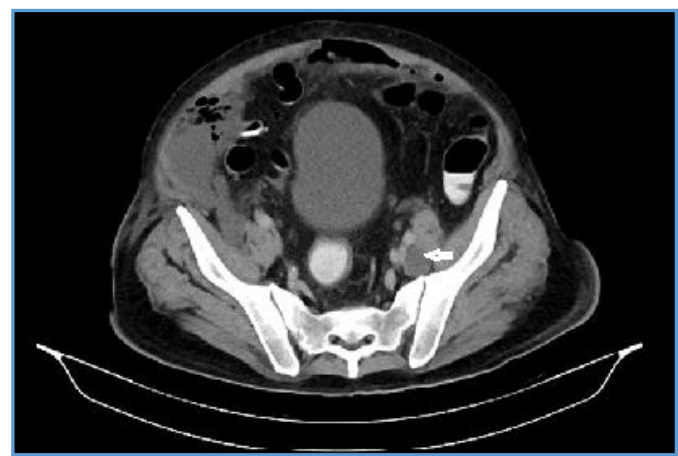

Figure 4. CECT W/A showing Collection postero-medial to Left Iliopsoas Muscle (Arrow)



Figure 5. CECT W/A showing Appendicular Tip (Arrow)

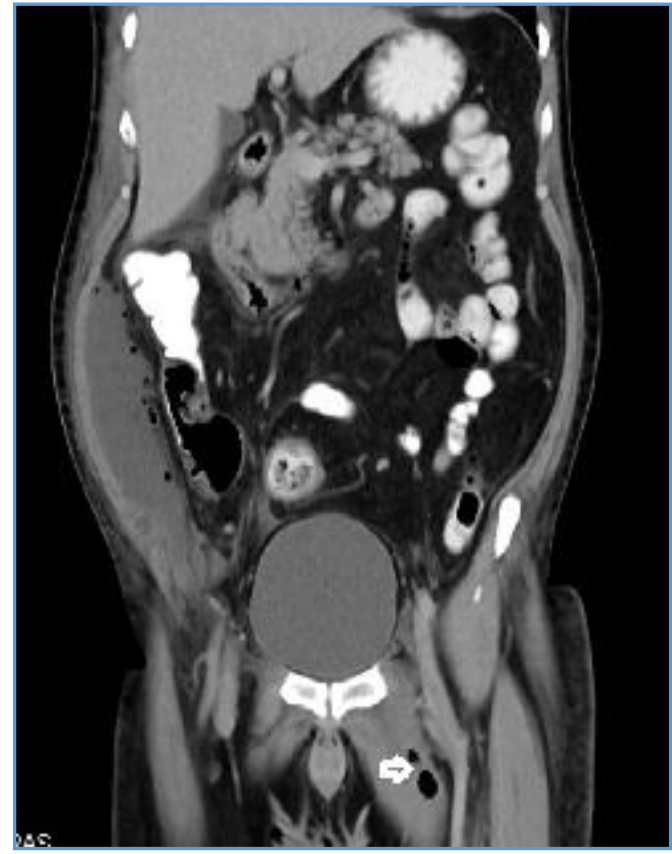

Figure 6. CECT W/A showing Collection with Air Pocket in Left Thigh (Arrow)

\section{Management}

Initially, patient was managed with supportive measures including moist $\mathrm{O}_{2}$ inhalation, nebulisation with Levosalbutamol and Budesonide respules, IV fluid, Inj. Pantoprazole $40 \mathrm{mg}$, infusion Paracetamol $1 \mathrm{gm}$ and Inj. Ceftriaxone $1 \mathrm{gm}$. But after receiving blood reports showing high total leucocyte count and high CRP and considering the patient's clinical condition, antibiotic was changed and Inj. Meropenem $1 \mathrm{gm}$ was started thrice daily.

On 26-02-2018, when CECT whole abdomen report was suggestive of suspected extraperitoneal rupture of appendicular tip, on the same day laparotomy was performed on emergency basis.

\section{Operative Findings}

- Large amount of pus was seen on right-sided extraperitoneal space and the extension of abscess cavity was from perinephric space above, right inguinal canal below and medially crossing the midline towards the left-sided retroperitoneal space.

- Intra-abdominally, small bowel loops were dilated, and ascites was seen. Besides appendix and ileal loop of 10 $\mathrm{cm}$ length, $15 \mathrm{~cm}$ proximal to ileocaecal junction were adhered to right-sided parietal peritoneum. Appendix 
was conglomerated with thickened mesoappendix. But there was no perforation in appendix.

\section{Procedures Performed}

- Drainage of retroperitoneal pus from both sides was done.

- Adhesiolysis of distal ileum along with appendectomy were done.

- Thorough search was done to exclude hollow viscus perforation.

- Thorough abdominal lavage was done with 3 - $4 \mathrm{~L}$ Normal Saline.

- Abdominal wall was repaired in 2 layers with keeping an abdominal drain in pelvis and 2 corrugated drains in both sided extraperitoneal spaces.

- Specimen of appendix was sent for histopathological examination and pus was sent for culture and sensitivity and TB-PCR.

\section{Post-Operative Period}

- Post-operative period was uneventful. On $1^{\text {st }}$ postoperative day, oral feeding was started. No fever was present from $2^{\text {nd }}$ post-operative day onwards. Antibiotics were kept as same as pre-operatively. On $3^{\text {rd }}$ post-operative day, intra-abdominal drain was removed as there was no drain output in last $48 \mathrm{hrs}$.

- Culture of pus showed growth of Klebsiella pneumonia and pus for TB PCR showed negative result. Histopathological examination of appendix showed inflammatory appendicitis.

- $\quad$ Patient was discharged in stable condition on $6^{\text {th }}$ postoperative day with the drains (placed extraperitoneally).

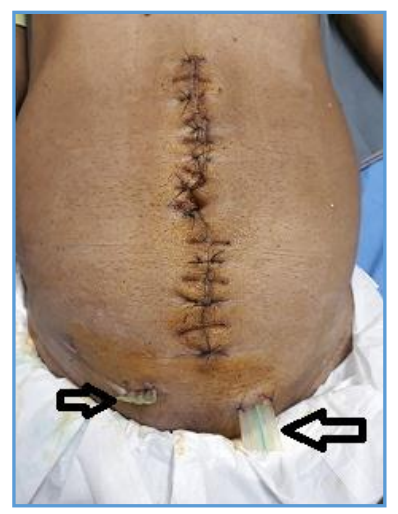

Figure 7. Picture of Patient during Discharge from hospital with Drains Placed Extraperitoneally (Arrows)

\section{DIFFERENTIAL DIAGNOSIS}

- Appendicitis (especially retrocaecal).

- Diverticulitis.

- Epidural Abscess.

- Intra-abdominal infection.

- Malignancy.

- Necrotising fasciitis.

- Osteomyelitis.

- Pancreatitis.

- Perforated bowel.

- Psoas abscess.

- Pyelonephritis.

- Retroperitoneal haematoma.

- $\quad$ Spinal TB.

\section{CLINICAL DIAGNOSIS}

Retroperitoneal abscess is a rare condition of infection and it is less common than intra-abdominal abscess. It mostly occurs in people aged 30 - 50 years with a slight male predominance. ${ }^{1}$ The clinical manifestation of retroperitoneal abscesses is undistinguished with common presentations of fever and pain.2,3 Therefore, initial misdiagnosis or delayed diagnosis is common. In many case series, fever, back pain and abdominal pain were common presentations. In the retrospective review by Crepps et al, ${ }^{3}$ it took an average of 12.7 days to establish a diagnosis. In suspected cases, a thorough examination should be done to exclude tender masses in the abdomen, flank and more rarely in the thigh, groin or scrotum. Most patients with retroperitoneal abscesses present non-specifically with malaise, fever, nausea and weight loss. ${ }^{4}$ Patients may present with extra-abdominal manifestations, for example referred pain to the hip or knee 4 or painful inguinal swellings. ${ }^{5}$ There have been various case reports describing abscesses in the retroperitoneum, which manifests distally in the thigh. ${ }^{6}$ On a literature review, there are many cases of retroperitoneal abscesses associated with retrocaecal appendicitis. ${ }^{7}$

\section{PATHOLOGICAL DISCUSSION}

The retroperitoneal space is a potential space between the peritoneum and the transversalis fascia lining the posterior abdominal cavity and extends from the diaphragm superiorly, pelvic brim inferiorly and margin of the quadratus lumborum muscles laterally. It is divided into three distinct compartments: the anterior pararenal, perirenal, and posterior pararenal spaces. The anterior pararenal space mainly contains digestive organs including the ascending and descending colon, pancreas and retroperitoneal part of the duodenum. The perirenal space contains the kidneys, adrenal glands, aorta and inferior vena cava. The posterior pararenal space contains fat with the spine and quadratus lumborum muscles located posteriorly. The space is closed superiorly in the diaphragm, but is open to the pelvis and thighs which causes abscesses to extend bilaterally and inferiorly. The pathogen of the retroperitoneal abscess may vary depending on the origin of the infection. Polymicrobial infections with aerobic and anaerobic bacteria were noted commonly in cases of GI origin and E. coli was the most common pathogen overall followed by $\mathrm{K}$. pneumoniae in many series. Mycobacteria was found to be the most common pathogen in abscesses originating from the spine, which was consistent with other series. ${ }^{2,3}$ The prevalence of K. pneumoniae rather than Proteus mirabilis was higher in abscesses of GU origin in Taiwan than in other countries. ${ }^{8}$

\section{DISCUSSION OF MANAGEMENT}

Contrast CT scan is reliable for the diagnosis of retroperitoneal abscess with a sensitivity ranging from $90 \%$ to $100 \%$. $^{2,3}$ It provides information on the extent of involvement and can assist in surgical planning.

MRI has also value for evaluation, especially in the abscesses originating from spinal origin for its clear vision of paraspinal soft tissues. ${ }^{9}$

Interventional treatment such as image-guided drainage or surgery is the mainstream treatment method along with antibiotics treatment. Even multiloculated abscesses can be treated with multiple drainage tubes or septal perforation 
with catheter placement. ${ }^{10}$ For perinephric abscesses and most psoas abscesses, percutaneous drainage along with prolonged antibiotic treatment was commonly administered initially with $10 \%$ of inadequate drainage rate which was still needed following surgical drainage. 2,11

Treatment of retroperitoneal abscesses originating from the spine is varied. Small and uncomplicated abscesses can be treated with medication alone. For large abscesses both percutaneous and surgical drainage were performed, but the timing of spinal fusion was related to the stability of the spine and the neurological manifestation.

The mortality of retroperitoneal abscesses depends on the patient's comorbidities. The mortality rate was reported to be $26 \%$ in a series in 1987 and $1.5 \%$ in a series in $2003.2,3$

In our patient, considering his clinical condition, and CECT whole abdomen finding suggestive of extraperitoneal rupture of appendix, surgical drainage was done. Although, no intra-abdominal hollow viscus perforation was found, laparotomy helped to diagnose the situation as well as saved his life by thorough drainage of pus at the same time.

\section{FINAL DIAGNOSIS}

Retroperitoneal abscess is an uncommon type of infection. Its insidious, non-specific clinical presentation due to lack of peritoneal signs can lead to mortality due to misdiagnosis. The source of infection may be primary or secondary. Primary infection is haematologically disseminated and usually located in the muscle or spine. Secondary infection by contrast is involved with the infection from organs within the space. Patients may report referred pain to the lower limb, painful swellings in the groin, or there may be evidence of subcutaneous emphysema on clinical examination. A clinician should always suspect an intra-abdominal pathology in any unwell patient who has presented with discomfort in the hip or thigh and painful groin swellings may indeed have something more sinister intra-abdominally despite lack of obvious signs.

\section{REFERENCES}

[1] Harris LF, Sparks JE. Retroperitoneal abscess. Case report and review of the literature. Dig Dis Sci 1980;25(5):392-5.

[2] Manjon CC, Sanchez TA, Lara PJD, et al. Retroperitoneal abscesses-analysis of a series of 66 cases. Scand J Urol Nephrol 2003;37(2):139-44.

[3] Crepps JT, Welch JP, Orlando R 3rd. Management and outcome of retroperitoneal abscesses. Ann Surg 1987;205(3):276-81.

[4] Lin C, Chou S, Wu H, et al. Gastrointestinal: retroperitoneal abscess caused by appendicitis. J Gastroenterol Hepatol 2007;22:278.

[5] Rivera-Herrera JL, Otheguy, JN, Nieves-Ortega J, et al. Painful inguinal mass: uncommon presentation of a retroperitoneal abscess. Bol Asoc Med P R 1991;83(9):402-3.

[6] Rotstein OD, Pruett TL, Simmons RL. Thigh abscess. An uncommon presentation of intraabdominal sepsis. Am J Surg 1986;151(3):414-8.

[7] Hsieh $\mathrm{CH}$, Wang $\mathrm{YC}$, Yang $\mathrm{HR}$, et al. Extensive retroperitoneal and right thigh abscess in a patient with ruptured retrocecal appendicitis: an extremely fulminant form of a common disease. World J Gastroenterol 2006;12(3):496-9.

[8] Brook I, Frazier EH. Aerobic and anaerobic microbiology of retroperitoneal abscesses. Clin Infect Dis 1998;26(4):938-41.

[9] Darouiche RO. Spinal epidural abscess. N Engl J Med 2006;355(19):2012-20.

[10] Lang EK. Renal, perirenal and pararenal abscesses: percutaneous drainage. Radiology 1990;174(1):10913.

[11] Yacoub WN, Sohn HJ, Chan S, et al. Psoas abscess rarely requires surgical intervention. Am J Surg 2008;196(2):223-7. 